\title{
Early palliative care and the opioid crisis: ten pragmatic steps towards a more rational use of opioids
}

\author{
Jan Gaertner ${ }^{1}$, Christopher Boehlke ${ }^{2}$, Charles B. Simone II $^{3}$, David Hui ${ }^{4}$ \\ ${ }^{1}$ Palliative Care Center Hildegard, Basel, Switzerland; ${ }^{2}$ Clinic for Palliative Medicine, Medical Center - University of Freiburg, Faculty of Medicine, \\ University of Freiburg, Freiburg, Germany; ${ }^{3}$ Department of Radiation Oncology, New York Proton Center, New York, NY, USA; ${ }^{4}$ Department of \\ Palliative Care, Rehabilitation and Integrative Medicine, MD Anderson Cancer Center, Houston, TX, USA \\ Contributions: (I) Conception and design: All authors; (II) Administrative support: None; (III) Provision of study materials or patients: None; (IV) \\ Collection and assembly of data: All authors; (V) Data analysis and interpretation: All authors; (VI) Manuscript writing: All authors; (VII) Final \\ approval of manuscript: All authors. \\ Correspondence to: PD Dr. Jan Gaertner. Palliative Care Center Hildegard, Basel, Switzerland, Sankt Alban Ring 151, 4002 Basel, Switzerland. \\ Email: jan.gaertner@pzhi.ch.
}

\begin{abstract}
In palliative care, opioids and other controlled drugs are among the most commonly used and important medications. Opioids are associated with significant risk of dependence and misuse. In many developed countries, there is an epidemic of prescription opioid misuse and overdose deaths. Palliative care has a critical role educating patients about the safe use of opioids, providing universal screening and close monitoring, and prescribing opioids appropriately balancing the risks and benefits. This is particularly important in the era of early palliative care, when patients have much longer survival and potentially greater risk of misuse while on chronic opioid therapy. Here, we provided a critical appraisal of opioid use in the context of opioid crisis and early palliative care. We also present a pragmatic 10-step approach for the judicious use of opioids.
\end{abstract}

Keywords: Opioid crisis; early palliative care; cancer pain; pain management

Submitted Oct 26, 2018. Accepted for publication Jul 31, 2019.

doi: 10.21037/apm.2019.08.01

View this article at: http://dx.doi.org/10.21037/apm.2019.08.01

\section{Introduction}

In late 2017, the United States (US) government declared a public health emergency due to steadily growing death counts attributed to the current "opioid crisis" (or "opioid epidemic") (1). It had become evident that the liberal and rather uncritical prescription of opioids to patients with chronic non-cancer pain led to devastating consequences for millions of patients and their families (2). At the same time, the palliative care needs of patients in early stages of incurable cancers and other diseases are increasingly recognized, including the need for adequate pain management (3). Many cancer patients seen by specialist palliative care teams are expected to live considerably longer than before in this era of immunotherapy, and some may even experience a rather "chronic" course of the disease (4).
Therefore, cancer and palliative care specialists increasingly find themselves in the midst of a dilemma: on the one hand, patients with pain demand access to potent analgesics such as opioids, and on the other hand, clinicians are very much aware of the potential harms of these drugs, especially for patients who are expected to live for many years and may require constant treatment of their pain throughout this period.

In the current special issue of the Annals of Palliative Medicine $(A P M)$ on "Clinical challenges and pitfalls of early palliative care: must know from other disciplines for palliative care specialist," we will provide state-of-theart practice recommendations from the field of addiction and behavioral medicine concerning screening for and handling of the risks of addiction (non-medical substance dependence). Because of the acuteness of the concerns 
around the use of opioids in early palliative care and in the absence of evidence-based-recommendations, the editors of the $A P M$ decided to provide early practice guidance already as part of this editorial.

\section{The past: the crusade against opiophobia}

For decades, the palliative care and cancer pain community struggled to overcome misperceptions concerning morphine and other strong opioids $(5,6)$. The public, as well as many physicians, associated criminal, immoral, hedonistic or even suicidal behavior with the use of these drugs (5). This stigma was not without reason, because especially in Europe, the intellectual elites and many physicians had massively abused opioids for decades as so called morphinists (or cocainists) in the early decades of the $20^{\text {th }}$ century $(7,8)$. Additionally, soldiers in World War II and the Vietnam War frequently abused opioids, which lead to disastrous consequences for the individuals but also their families continuing after their return from the war $(7,8)$. Opium had also been an everyday drug in the working class for what we would consider "chemical coping," reflected by Karl Marx's famous comparison that "religion is the opium for the people." (9). In addition to issues of abuse, in the cold war, "deadly doses of morphine" were suggested in the event of nuclear attacks, which drew public attention towards the "deadly properties" of morphine and other opioids (10). Over time, the pendulum swung towards opiophobia, with many clinicians, patients and families concerned about opioid use even when appropriate.

The palliative care, hospice and cancer pain communities worked hard to overcome the stigmas related to the use of morphine to make it available for those who truly and desperately needed it as a highly effective analgesic in the case of moderate to severe pain that could be directly from their cancer or from the therapies intended to treat their cancer (11). Their vigorous and often emotional crusade to promote access and use of opioids was driven by the experience that particularly in patients suffering from severe cancer pain opioids were massively underused because off physician ignorance, insufficient expertise, or failure to recommend this treatment $(5,11,12)$.

The campaign finally let to an alliance of scientific associations, healthcare authorities and the pharmaceutical industry (1). This is highlighted for example by the $5^{\text {th }}$ vital sign campaign of the International Association for the Study of Pain (IASP) (1), private public partnerships with the pharmaceutical industry (13) and the mantra of some key opinion leaders that "nobody has to suffer pain" (14).

As a result of these campaigns, the community even introduced having "average prescription of opioids per 1 million inhabitants" as a measured quality indicator for pain management $(15,16)$. In co-operation with pharmaceutical companies, so called "pain free hospitals" or even "pain free cities" were demanded, suggesting that being free of pain would just rely on adequate opioid doses $(13,17)$.

\section{The present: consequences of opiophilia}

As a result of the originally well-intentioned and reasonable campaign described above, the availability and prescription of opioids increased considerably over time. Meanwhile, in developed countries patients with advanced diseases have access to opioids in cases of moderate to severe pain, although training in and awareness for pain management in many physicians is far from being optimal $(6,16)$. Despite the quadrupling of prescribed opioid sales in the US, there has not been a change in the overall pain Americans report (18).

However, with the pendulum swinging from opiophobia to opiophilia in some settings, some physicians have adopted a rather liberal attitude of opioid prescribing, disregarding the potential harms especially for patients with substantial life expectancies or those who are at risk for addiction (non-medical substance dependence) $(1,2)$. For example, in Canada, $18.7 \%$ of non-palliative care patients receiving opioids for chronic non-cancer pain exceeded doses of $400 \mathrm{mg}$ morphine equivalent per day (19). Similarly, Morasco et al. (20) reported from a US cohort of non-palliative care patients with chronic non-cancer pain that a considerable number of patients on opioid-therapy (8.4\%) received high doses: in the high dose group, the morphine equivalent was $324.9 \mathrm{mg} /$ day ( $\mathrm{SD}=285.1)$ (20). Also, in these patients, prescriptions of non-sustained (immediate- or fast release-) formulations and also benzodiazepines (30\%) further aggravated the potential harms concerning addiction, diversion and mortality (20). Some opioid advocates from the pain and palliative care community who were acting as advisors of the Food Drug Administration (FDA) and lobbyists even launched trials using rapid onset (RO) fentanyl for the treatment of chronic low back pain (21-23).

In the meantime, opioid-associated deaths for example in the US increased four-fold from 1999 to 2008 and continued to rise thereafter, finally leading to the US government declaration of a public health emergency in 
late 2017 (20,24-26).

\section{Early palliative care and the prescription of opioids: pragmatic guidance for palliative care teams}

With the absence of evidence-based recommendations, and remembering the words of William Osler that "Medicine is a science of uncertainty and an art of probability" and that "even the best of men must be content with fragments, with partial glimpses," we propose the following 10 pragmatic steps as backbone recommendations for the use of opioids in early palliative care:

\section{Step 1: admit the problem}

Fortunately, in the $21^{\text {st }}$ century we no longer regard pain as an existential phenomenon of divine cause (27). Patients demand access to adequate pain management including the provision of opioids as their "human right" $(28,29)$.

At the same time, our frequently heard palliative care mantra "Neither addiction nor mortality issues are of relevance for opioid use" is a result of persisting re-iteration of arguments conveyed by key opinion leaders and lobbyists that were based on low-quality evidence only $(1,22,30,31)$. Addiction is always a potential concern for opioids, although some patients may be at higher risk than others.

\section{Step 2: understand the neurobiology of non-nociceptive systems}

Opioids commonly act via activation of mu receptors along the nociceptive pathway, finally resulting in diminished pain perception at the somatosensory cortex. Yet, mu receptors are also expressed in non-nociceptive structures of the brain and other parts of the body, leading to wellknown side effects such as nausea, somnolence and constipation. Especially via the limbic system, where a large concentration of mu receptors is located, they convey positive emotions such as delight, gratification and reward. The latter is a central component for the development of substance dependence (formerly called "addiction"). While analgesic tolerance along the nociceptive system develops slowly, tolerance for delight and other positive limbic sensations is acquired much earlier. Therefore, reduction of mu-associated limbic activity provokes dysphoria. Opioids also inhibit the locus caeruleus, which is part of the activating reticular system and the largest group of noradrenergic neurons in the central nervous system (32). Thus, mu-receptor tachyphylaxis and receptor downregulation results in symptoms of sympathetic rebound (33). It is also of importance to note that especially immediate release (IR), intravenous (IV) or RO opioids induce substantial reward mechanisms as well as faster tolerance on the non-nociceptive pathway. Thus, such forms of application demand extreme caution in patients with a long life-expectancy and those at risk for non-medical substance dependence (see below: Step 5)

\section{Step 3: screening: identify patients at risk}

Universal screening for addiction risk can help clinicians to tailor the monitoring strategy. The current psychiatric classification system (DSM-5) uses the term "substance dependence" instead of the older and more common word "addiction," partly in an attempt to decrease stigmatization (34). The change of terms inadvertently resulted in decreased clarity of the underlying concepts, especially when referring to the use of potentially therapeutic substances (i.e., opioids) (34). For example, with the new definition, physicians can diagnose 'opioid dependence' based exclusively on symptoms related to tolerance, physical dependence and withdrawal (34). Such criteria may be helpful for the diagnosis of alcoholism or heroin addiction, but in the case of opioid therapy for cancer pain, they result in false-positive identification of "abusers" and "addicts" (34).

Instead, the "four Cs" have been proposed by the Liaison Committee on Pain and Addiction (LCPA) of the American Academy of Pain Medicine (AAPM), the American Pain Society (APS) and the American Society of Addiction Medicine (ASAM),

(I) Impaired Control over drug use;

(II) Compulsive use;

(III) Continued use despite harm (Consequences);

(IV) Craving.

No single "C" is diagnostic, and the diagnosis should be made from a pattern of behavior over time (35).

Also, the CAGE-AID questions for the use of both alcohol and illicit drugs are frequently recommended (34):

In the past, have you ever:

- Tried to Cut down or Change your pattern of drinking or drug use?

* Been Annoyed by others' concerns about your drinking or drug use?

* Felt Guilty about the consequences of your drinking or 
drug use?

* Had a drink or used a drug in the morning (Eyeopener) to decrease hangover or withdrawal symptoms?

One positive response to any of the CAGE-AID questions would suggest caution. Two or more positive responses require further assessment for a serious alcohol or drug problem.

Also, other behavior may suggest problematic use of opioids. Examples include (36):

* Unscheduled visits, phone calls or emergency admissions for opioids;

* Self-escalation of opioid dose or usage frequency;

* High or extreme pain intensity with the absence of other symptoms or distress;

* Reports of opioids lost or stolen, discrepancies in pill counts;

* Formal or informal care givers expressing concern over the patient's opioid use.

In all patients at risk for problematic opioid use, drug screening (urine) should also be considered.

\section{Step 4: tapering: do not forget to stop opioids whenever possible}

Although it is important to initiate opioid therapy in the early stages of the disease or even in curative settings, in case it is indicated, it must also be tapered and stopped when pain relief is achieved. For patients presenting with pain that is expected to resolve within a certain timeframe, it is important to discuss the goal and duration of opioid therapy upfront to set expectations. For example, Kwon et al. (37) reported from a cohort of patients who were receiving opioids due to pain from curative radiotherapy for head and neck cancer that one of three patients was still on opioids half a year later and that many of these patients were CAGE-AID positive. Thus, it is mandatory to schedule regular "opioid-tapering visits" for these patients to prevent unnecessary chronification of opioid therapy.

\section{Step 5: monitor over time and consider further surveillance measures}

If tapering is inadequate or unfeasible, "monitoring and mitigation visits" should be scheduled as for example in the treatment of chronic pain in cancer survivors (38). Here, indicators for aberrant opioid consumption should be actively assessed alongside the view of family members concerning the opioid (ab-)use pattern of the patient (38).
If aberrant behavior is observed and the benefits of opioid therapy outweigh the risks, measures to increase opioid surveillance should be implemented if the decision is to continue therapy (38). For example, only small amounts of opioids should be prescribed at short intervals and written "contracts" with the patient should be pursued (38). Also, pill counts should be mandatory, but of course, obtaining opioid prescriptions from multiple prescribers/pharmacies around the same time ("doctor shopping") cannot be avoided completely, leaving much room for unobserved aberrant behaviour (39). Therefore, many regions have implemented drug monitoring programs for opioids and other controlled drugs to support physicians in the detection of opioid abuse issues, but pharmacists often do not feel adequately trained (40) and the development of national programs is advocated to overcome regional disparities (39). Nonetheless, in patients at risk, urine screening for opioids and other substances should be performed routinely $(41,42)$.

\section{Step 6: consider alternative interventions and be aware of the psycho-social aspects of pain}

Especially in the early, often perioperative setting, the provision of regional anesthesia techniques might be helpful to avoid IV PCA. Also, non-opioid analgesics and coanalgesics against neuropathic pain should be considered, although the use of pregabalin and other pentanoids can also be associated with non-medical substance dependency issues (43). In cases of chemotherapy-induced painful neuropathy, capsaicin applied locally, acupuncture and antidepressants such as duloxetine or amitriptyline (given as a co-analgesic against the neuropathic pain component, rather than as antidepressants) may be helpful.

It has long been known that pain has relevant psychosocial and spiritual components (total pain) (44). Sometimes, these non-physical dimensions are even the main contributor to pain or pain perception. This means that sufficient pain management must not rely solely on the prescription of drugs (i.e., opioids). Instead, close attention must be paid to other causes of suffering. Especially in patients when the initial pharmaceutical pain management efforts are ineffective, the psycho-social dimension of suffering demands close attention. Here, cooperations between cancer-specialists, psycho-oncologists, palliative care specialists and pain services are of the utmost importance to navigate the patients and ensure the provision of multidisciplinary biopsychosocial rehabilitation, cognitive behavioral therapy, mindfulness-based therapy, relaxation 
techniques, biofeedback, distraction techniques and other interventions if indicated (45-47).

\section{Step 7: be cautions with rapid onset formulations}

As described above, RO or IR formulations and IV injections result in pronounced limbic activity ("reward"). This results in a more than fourfold potential of abuse and addiction compared to sustained release (SR) opioids (48). Yet, it must not be overlooked that SR opioids instead carry a higher risk of fatal overdose (49).

Although the World Health Organization recommendations for cancer pain management call for opioids "by the need" to treat pain episodes, SR or transdermal opioid formulations are generally preferred because they allow a more proactive approach to pain management and may be associated with lower risk of opioid-dependence. RO fentanyl formulations (buccal/nasal) and also IV patient-controlled analgesia (PCA) should be used for very specific indications and may not be appropriate for many patients.

Some authors have also promoted the preferred use of methadone or the partial antagonist buprenorphine in these patients (36). Yet, the true effects of this approach remain unclear.

\section{Step 8: establish structured interdisciplinary collaborations}

Especially for patients at risk for non-medical opioiddependence and whenever complex psycho- social problems are suspected, close co-operations between cancerspecialists, psycho-oncology, specialist palliative care, addictions specialists, chronic and interventional pain services are of the utmost importance as described above $(50,51)$. To establish an effective network that meets the increasing challenges in light of the opioid crisis, workshops with the different teams may help to develop a joint agenda and a common understanding of best practices and referral criteria (who, when, why, what?).

\section{Step 9: start educating patients and staff about the safe use, storage, and disposal of opioids}

Whenever opioids are prescribed, physicians should consider the patients' social environment to reduce substance abuse. Many patients, but also clinical providers, do not have adequate knowledge and awareness of how to prevent family members or others to access the patients' medication (52). Patients should be educated to never share their medications with others and not to take opioids or other psychotropic medications not prescribed for them. Simple education material have been found to improve patients' understanding of safe opioid use, disposal and storage (52). As a minimum, patients should be informed

* To avoid getting opioids from multiple teams of prescribers (52);

* To abstain from talking publicly about the controlled medication they use (52);

* To abstain from sharing or borrowing their medications (52);

* To keep the opioid medications stored in a safe and secured place (52);

* To minimize using opioids with other psychotropic agents (52);

* To dispose of medications via normal trash (mixed with cat litter or coffee grounds to make them undesirable) or flush them down the toilet (52).

\section{Step 10: continue fighting opiophobia}

The critical look on the use of opioids, especially in early palliative care, must not lead to a relapse of opiophobia. This means that depending on the attitudes and expertise of their physicians, some patients with severe (i.e., cancer) pain, but also with dyspnea are still at risk for not receiving opioid therapy as it would be mandatory to achieve adequate relief of suffering from their symptoms $(6,53)$. Also, the opioid crisis and a more critical view on the potential harms of opioids have already led to decreased opioid provision in patients for whom such restraints are not at all adequate (54). For example, a working group from the MD Anderson Cancer Center reported that the median opioid daily opioid dose upon referral of patients referred to the palliative care clinic almost halved over a five-year period, suggesting that oncologists may be less comfortable prescribing opioids (54).

\section{Conclusions}

Before initiation of opioid therapy in patients in early stages of incurable disease and especially in potential long-term "survivors," a number of critical considerations should be made. With the 10 steps suggested in this article, the authors and editors hope to provide pragmatic guidance for palliative care teams, cancer specialists and other disciplines for a more deliberate approach of providing pain relief with and without opioids in the light of the current opioid crisis. 
It did not escape our attention, that in many parts of the world, the situation is completely different and opioids are still insufficiently available even for patients with severe pain from advanced cancer (55).

\section{Acknowledgments}

None.

\section{Footnote}

Conflicts of Interest: The author has no conflicts of interest to declare.

Ethical Statement: The authors are accountable for all aspects of the work in ensuring that questions related to the accuracy or integrity of any part of the work are appropriately investigated and resolved.

\section{References}

1. Jones MR, Viswanath O, Peck J, et al. A Brief History of the Opioid Epidemic and Strategies for Pain Medicine. Pain Ther 2018;7:13-21.

2. Comerci G Jr, Katzman J, Duhigg D. Controlling the Swing of the Opioid Pendulum. N Engl J Med 2018;378:691-3.

3. Ferrell BR, Temel JS, Temin S, et al. Integration of Palliative Care Into Standard Oncology Care: American Society of Clinical Oncology Clinical Practice Guideline Update. J Clin Oncol 2017;35:96-112.

4. Farkona S, Diamandis EP, Blasutig IM. Cancer immunotherapy: the beginning of the end of cancer? BMC Med 2016;14:73.

5. Zenz M, Willweber-Strumpf A. Opiophobia and cancer pain in Europe. Lancet 1993;341:1075-6.

6. Breuer B, Fleishman SB, Cruciani RA, et al. Medical oncologists' attitudes and practice in cancer pain management: a national survey. J Clin Oncol 2011;29:4769-75.

7. Tischler V. Dr Junkie. The Doctor Addict in Bulgakov's Morphine: What are the Lessons for Contemporary Medical Practice? J Med Humanit 2015;36:359-68.

8. Walter H. "Epidemic" or Peripheral Phenomenon?: A Medical History of the "Cocaine Wave" in the Weimar Republic. NTM 2017;25:311-48.

9. Sabatowski R, Schäfer D, Kasper SM, et al. Pain treatment: a historical overview. Curr Pharm Des 2004;10:701-16.
10. Two British MDs favor lethal dose of morphine in case of nuclear attack. Med World News 1984;25:72.

11. Simone CB 2nd, Vapiwala N, Hampshire MK, et al. Cancer patient attitudes toward analgesic usage and pain intervention. Clin J Pain 2012;28:157-62.

12. Jeon YS, Kim HK, Cleeland CS, et al. Clinicians' practice and attitudes toward cancer pain management in Korea. Support Care Cancer 2007;15:463-9.

13. Osterbrink J, Ewers A, Nestler N, et al. Health services research project "action alliance pain-free city Münster" : Objectives and methods." Schmerz. 2010;24:613-20.

14. Available online: https://www.stern.de/gesundheit/ therapie-den-schmerz-beherrschen-3078062.html

15. Nakazawa $Y$, Kato M, Yoshida S, et al. Population-Based Quality Indicators for Palliative Care Programs for Cancer Patients in Japan: A Delphi Study. J Pain Symptom Manage 2016;51:652-61.

16. Linge-Dahl L, Vranken $M$, Juenger $S$, et al. Identification of Challenges to the Availability and Accessibility of Opioids in Twelve European Countries: Conclusions from Two ATOME Six-Country Workshops. J Palliat Med 2015;18:1033-9.

17. Ripamonti C, Valle A, Peruselli C, et al. Project "Hospital without pain": analysis of the Italian situation before the law 38. Assist Inferm Ric 2011;30:95-9.

18. Chang HY, Daubresse M, Kruszewski SP, et al. Prevalence and treatment of pain in EDs in the United States, 2000 to 2010. Am J Emerg Med 2014;32:421-31.

19. Spooner L, Fernandes K, Martins D, et al. High-Dose Opioid Prescribing and Opioid-Related Hospitalization: A Population-Based Study. PLoS One 2016;11:e0167479.

20. Morasco BJ, Duckart JP, Carr TP, et al. Clinical characteristics of veterans prescribed high doses of opioid medications for chronic non-cancer pain. Pain 2010;151:625-32.

21. Portenoy RK, Messina J, Xie F, et al. Fentanyl buccal tablet (FBT) for relief of breakthrough pain in opioidtreated patients with chronic low back pain: a randomized, placebo-controlled study. Curr Med Res Opin 2007;23:223-33.

22. Whoriskey P. Rising painkiller addiction shows damage from drugmakers' role in shaping medical opinion. Available online: https://www.washingtonpost.com/ business/economy/2012/12/30/014205a6-4bc3-11e2b709-667035ff9029_story.html?noredirect=on\&utm_ term $=. d f a a 72 \mathrm{c} 10 \mathrm{bb} 2$

23. Haffajee RL, Mello MM. Drug Companies' Liability for the Opioid Epidemic. N Engl J Med 2017;377:2301-5. 
24. Jones GH, Bruera E, Abdi S, et al. The Opioid Epidemic in the United States-Overview, Origins, and Potential Solutions. Cancer 2018;124:4279-86.

25. Haffajee RL, Frank RG. Making the Opioid Public Health Emergency Effective. JAMA Psychiatry 2018;75:767.

26. Haffajee R, Parmet WE, Mello MM. What Is a Public Health "Emergency"? N Engl J Med 2014;371:986-8.

27. Meldrum ML. A capsule history of pain management. JAMA 2003;290:2470-5.

28. James A. Painless human right. Lancet (London, England) 1993;342:567-8.

29. Adams V, Worldwide Palliative Care Alliance. Access to pain relief: an essential human right. A report for World Hospice and Palliative Care Day 2007. Help the hospices for the Worldwide Palliative Care Alliance. J Pain Palliat Care Pharmacother 2008;22:101-29.

30. Portenoy RK, Foley KM. Chronic use of opioid analgesics in non-malignant pain: report of 38 cases. Pain 1986;25:171-86.

31. Porter J, Jick H. Addiction rare in patients treated with narcotics. N Engl J Med 1980;302:123.

32. Samuels ER, Szabadi E. Functional neuroanatomy of the noradrenergic locus coeruleus: its roles in the regulation of arousal and autonomic function part II: physiological and pharmacological manipulations and pathological alterations of locus coeruleus activity in humans. Curr Neuropharmacol 2008;6:254-85.

33. Scherbaum N, Bonnet U. Neurobiology of opioid dependence. Schmerz 2018;32:483-94.

34. Jovey RD. Opioids, pain and addiction - practical strategies. Br J Pain 2012;6:36-42.

35. American Academy of Pain Medicine, the American Pain Society and the American Society of Addition Medicine. Definitions related to the use of opioids for the treatment of pain. WMJ 2001;100:28-9.

36. Bruera E, Del Fabbro E. Pain Management in the Era of the Opioid Crisis. Am Soc Clin Oncol Educ book Am Soc Clin Oncol Annu Meet 2018;38:807-12.

37. Kwon JH, Hui D, Chisholm G, et al. Predictors of longterm opioid treatment among patients who receive chemoradiation for head and neck cancer. Oncologist 2013;18:768-74.

38. Paice JA, Portenoy R, Lacchetti C, et al. Management of chronic pain in survivors of adult cancers: American Society of Clinical Oncology clinical practice guideline. J Clin Oncol 2016;34:3325-45.

39. D'Souza RS, Eldrige JS. Prescription Drug Monitoring Program. StatPearls [Internet]. Treasure Island (FL):
StatPearls Publishing; 2019 Jul 14.

40. Thakur T, Frey M, Chewning B. Pharmacist Services in the Opioid Crisis: Current Practices and Scope in the United States. Pharmacy 2019;7:60.

41. Arthur JA, Haider A, Edwards T, et al. Aberrant Opioid Use and Urine Drug Testing in Outpatient Palliative Care. J Palliat Med 2016;19:778-82.

42. Arthur JA, Edwards T, Lu Z, et al. Frequency, predictors, and outcomes of urine drug testing among patients with advanced cancer on chronic opioid therapy at an outpatient supportive care clinic. Cancer 2016;122:3732-9.

43. Gaertner J, Schiessl C. Cancer pain management: what's new? Curr Pain Headache Rep 2013;17:328.

44. Clark D. "Total pain", disciplinary power and the body in the work of Cicely Saunders, 1958-1967. Soc Sci Med 1999;49:727-36.

45. Swedish Council on Health Technology Assessment. Methods of Treating Chronic Pain: A Systematic Review. SBU Yellow. 2006.

46. Marin TJ, Van Eerd D, Irvin E, et al. Multidisciplinary biopsychosocial rehabilitation for subacute low back pain. Cochrane Database Syst Rev 2017;6:CD002193.

47. Garland EL, Froeliger B, Howard MO. Effects of Mindfulness-Oriented Recovery Enhancement on reward responsiveness and opioid cue-reactivity. Psychopharmacology (Berl) 2014;231:3229-38.

48. Iwanicki JL, Severtson SG, McDaniel H, et al. Abuse and Diversion of Immediate Release Opioid Analgesics as Compared to Extended Release Formulations in the United States. PLoS One 2016;11:e0167499.

49. Miller M, Barber CW, Leatherman S, et al. Prescription opioid duration of action and the risk of unintentional overdose among patients receiving opioid therapy. JAMA Intern Med 2015;175:608-15.

50. Hui D, Hannon BL, Zimmermann C, et al. Improving patient and caregiver outcomes in oncology: Team-based, timely, and targeted palliative care. CA Cancer J Clin 2018;68:356-76.

51. Hui D, Mori M, Watanabe SM, et al. Referral criteria for outpatient specialty palliative cancer care: an international consensus. Lancet Oncol 2016;17:e552-9.

52. Reddy A, de la Cruz M, Rodriguez EM, et al. Patterns of Storage, Use, and Disposal of Opioids Among Cancer Outpatients. Oncologist 2014;19:780-5.

53. Ahmadi Z, Bernelid E, Currow D, et al. Prescription of opioids for breathlessness in end-stage COPD: a national population-based study. Int J Chron Obstruct Pulmon Dis 2016;11:2651-7. 
54. Haider A, Zhukovsky DS, Meng YC, et al. Opioid Prescription Trends Among Patients With Cancer Referred to Outpatient Palliative Care Over a 6-Year Period. J Oncol Pract 2017;13:e972-81.

55. Pastrana T, Wenk R, Radbruch L, et al. Pain Treatment

Cite this article as: Gaertner J, Boehlke C, Simone CB 2nd, Hui D. Early palliative care and the opioid crisis: ten pragmatic steps towards a more rational use of opioids. Ann Palliat Med 2019;8(4):490-497. doi: 10.21037/apm.2019.08.01
Continues To Be Inaccessible for Many Patients Around the Globe: Second Phase of Opioid Price Watch, a CrossSectional Study To Monitor the Prices of Opioids. J Palliat Med 2017;20:378-87. 